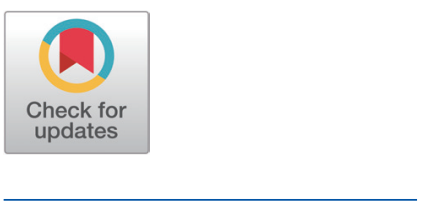

Received: Jun 3, 2021

Revised: Jul 20, 2021

Accepted: Jul 27, 2021

*Corresponding author

Dae-Kyung Kang

Department of Animal Resources

Science, Dankook University, Cheonan

31116, Korea.

Tel: +82-41-550-3655

E-mail: dkkang@dankook.ac.kr

Copyright $\odot 2021$ Korean Society of Animal Sciences and Technology.

This is an Open Access article distributed under the terms of the Creative Commons Attribution

Non-Commercial License (http:// creativecommons.org/licenses/bync/4.0/) which permits unrestricted non-commercial use, distribution, and reproduction in any medium, provided the original work is properly cited.

ORCID

Ju Kyoung Oh

https://orcid.org/0000-0002-8554-1863

Robie Vasquez

https://orcid.org/0000-0002-2878-9125

Sang Hoon Kim

https://orcid.org/0000-0001-9811-2972

In-Chan Hwang

https://orcid.org/0000-0001-8268-989X Ji Hoon Song

https://orcid.org/0000-0003-0027-7416

Jae Hong Park

https://orcid.org/0000-0002-2025-0141

In Ho Kim

https://orcid.org/0000-0003-2135-8098

Dae-Kyung Kang

https://orcid.org/0000-0001-9241-1250

\section{Multispecies probiotics alter fecal short-chain fatty acids and lactate levels in weaned pigs by modulating gut microbiota}

\author{
Ju Kyoung Oh${ }^{1}$, Robie Vasquez ${ }^{2}$, Sang Hoon Kim², In-Chan Hwang ${ }^{2}$, \\ Ji Hoon Song ${ }^{2}$, Jae Hong Park², In Ho Kim² and Dae-Kyung Kang ${ }^{2 *}$ \\ ${ }^{1}$ Department of Microbiology, Tumor and Cell Biology, Centre for Translational Microbiome Research (CTMR), \\ Karolinska Institutet, Stockholm 17177, Sweden \\ ${ }^{2}$ Department of Animal Resources Science, Dankook University, Cheonan 31116, Korea
}

\section{Abstract}

Short-chain fatty acids (SCFAs) are metabolic products produced during the microbial fermentation of non-digestible fibers and play an important role in metabolic homeostasis and overall gut health. In this study, we investigated the effects of supplementation with multispecies probiotics (MSPs) containing Bacillus amyloliquefaciens, Limosilactobacillus reuteri, and Levilactobacillus brevis on the gut microbiota, and fecal SCFAs and lactate levels of weaned pigs. A total of 38 pigs weaned at 4 weeks of age were fed either a basal diet or a diet supplemented with MSPs for 6 weeks. MSP administration significantly increased the fecal concentrations of lactate (2.3-fold; $p<0.01$ ), acetate (1.8-fold; $p<0.05$ ), and formate (1.4-fold; $p$ $<0.05$ ). Moreover, MSP supplementation altered the gut microbiota of the pigs by significantly increasing the population of potentially beneficial bacteria such as Olsenella, Catonella, Catenibacterium, Acidaminococcus, and Ruminococcaceae. MSP supplementation also decreased the abundance of pathogenic bacteria such as Escherichia and Chlamydia. The modulation of the gut microbiota was observed to be strongly correlated with the changes in fecal SCFAs and lactate levels. Furthermore, we found changes in the functional pathways present within the gut, which supports our findings that MSP modulates the gut microbiota and SCFAs levels in pigs. The results support the potential use of MSPs to improve the gut health of animals by modulating SCFAs production.

Keywords: Short-chain fatty acid, Lactate, Multispecies probiotic, Gut microbiota, Probiotic, Weaned pig

\section{INTRODUCTION}

Short-chain fatty acids (SCFAs) are metabolic products produced during the microbial fermentation of non-digestible fibers [1-3]. The fermentation of amino acids also contributes to the production of SCFAs $[3,4]$. They are produced at high levels by bacteria in the proximal colon, where they are consumed by enterocytes [5]. The major SCFAs produced in the gut are formate, acetate, butyrate, 
Competing interests

No potential conflict of interest relevant to this article was reported.

\section{Funding sources}

This work was supported by the Korea Institute of Planning and Evaluation for Technology in Food, Agriculture, Forestry and Fisheries (IPET) through The Strategic Initiative for Microbiomes in Agriculture and Food, funded by Ministry of Agriculture, Food and Rural Affairs (MAFRA) (918001-04-4 WT011). In addition, the present research was supported by the research fund of Dankook University in 2019 for the University Innovation Support Program.

Acknowledgements

Not applicable.

Availability of data and material Upon reasonable request, the datasets of this study can be available from the corresponding author.

Authors' contributions

Conceptualization: Park JH, Kim IH, Kang DK

Data curation: Oh JK, Vasquez R.

Formal analysis: Oh JK, Vasquez R.

Methodology: Oh JK, Park JH, Kim IH, Kang

DK.

Software: Oh JK, Kim SH

Investigation: Oh JK, Vasquez R, Kim SH, Hwang IC, Song JH.

Visualization: Vasquez $\mathrm{R}$.

Writing - original draft: Oh JK, Vasquez R.

Writing - review \& editing: Oh JK, Vasquez R, Kang DK.

Ethics approval and consent to participate All animal protocols were approved by the Dankook University Animal Care Committee (DK-2-2018). and propionate, while lactate and other organic acids are produced as well [1,3]. The production of SCFAs involves a complex enzymatic process, commonly involving glycolysis, or, in other bacteria, pentose phosphate pathway [5]. Certain species of gut bacteria also facilitates metabolic crossfeeding, wherein, an SCFA (such as acetate or lactate) is converted into another SCFA (such as butyrate) $[5,6]$. SCFAs play an important role in metabolic homeostasis and overall gut health in humans and animals. These metabolites are known to improve gut integrity, regulate glucose and lipid homeostasis, and improve immune function [1,2].

Host diets play a crucial role in determining the level of SCFAs produced by gut microbiota $[7,8]$. Diets rich in fibers or supplemented by prebiotics can trigger the production of high levels of SCFAs $[7,9,10]$. In addition, previous studies have shown the ability of probiotics to modulate SCFA production, with regard to host health [11,12]. Dietary supplementation with a combination of microbial species, herein called multispecies probiotics (MSPs), was beneficial due to improved growth performance, reduction of incidence of diarrhea, and protection against pathogens in animals [13-16]. Although the modulatory effects of MSPs on host gut microbiota has been also examined in previous studies, there is still limited information on its effect on the modulation of gut microbiota and production of SCFAs in pigs, which is economically important in animal industry. Therefore, the goal of this study is to investigate the effects of MSP supplementation on the gut microbiota and fecal SCFA and lactate levels of weaned pigs. We also aim to examine the relationship between the gut microbiota and the levels of fecal SCFA after MSP feeding.

\section{MATERIALS AND METHODS}

\section{Animal diets and experimental design}

A total of 38 pigs (Landrace $\times$ Yorkshire $\times$ Duroc) were used in this study, which were weaned at 4 weeks of age and had an average initial body weight of $23.53 \pm 0.12 \mathrm{~kg}$. The pigs were randomly divided into two groups: 18 pigs were placed in the control (CON) group, and the other 20 pigs in the MSP group. Each pen contained five pigs and was equipped with a one-sided self-feeder and nipple waterer for ad libitum access to feed and water. For 6 weeks, the control group continuously received the basal diet (Supplementary Table S1) without the administration of antibiotics or probiotics, while the probiotic (MSP) group received the basal diet supplemented with MSPs containing Bacillus amyloliquefaciens G10 (National Center for Biotechnology Information [NCBI] accession number CP072612), Levilactobacillus brevis M10 (NCBI accession number CP079699), and Limosilactobacillus reuteri RTR (NCBI accession number CP073062). These microbial species selected for the MSP supplement were isolated in a previous study for their potential probiotic characteristics in vitro (unpublished data). Individual probiotics were prepared using solid-state fermentation process, then processed into powdered form. The powdered probiotics were mixed with the basal feed to achieved their individual dosages. These processes were performed in the Genebiotech (Seoul, Korea). The dosages of B. amyloliquefaciens G10, L. brevis M10, and L. reuteri RTR added to the feed of the MSP group were $2.8 \times 10^{8} \mathrm{CFU} / \mathrm{g}$ feed, $2.3 \times 10^{7} \mathrm{CFU} / \mathrm{g}$ feed, and $4.8 \times 10^{6} \mathrm{CFU} / \mathrm{g}$ feed, respectively, and the viable cell counts were confirmed on the day of feeding. Fresh fecal samples were collected from the rectum of each pig before and after treatment, then stored at $-20^{\circ} \mathrm{C}$ until further analyses. All animal protocols were approved by the Dankook University Animal Care Committee.

\section{Fecal short-chain fatty acids and lactate concentration measurements}

Fecal samples were prepared for high-performance liquid chromatography (HPLC) analyses as previously described [17]. The concentrations of SCFAs (formate, acetate, propionate, and butyrate) 
and lactate in pig fecal samples were determined via HPLC following the method described by De Baere et al. [18] with modifications. Briefly, $10 \mathrm{mM}$ potassium dihydrogen phosphate $(\mathrm{pH} 2.2)$ and methanol (80:20 ratio) were used as the mobile phase with a C-18 reversed phase $(150 \times 4.6$ $\mathrm{mm}, 5 \mu \mathrm{m}$ ) column (Young Jin Biochrom, Seongnam, Korea). HPLC was performed using a 1260 Infinity HPLC machine (Agilent, Santa Clara, CA, USA) equipped with a quaternary pump set, autosampler, column thermostat set at $35^{\circ} \mathrm{C}$, and a multi-wavelength detector set at $210 \mathrm{~nm}$.

\section{S rRNA sequencing and microbial community analyses}

Genomic DNA was extracted from the fecal samples using a QiaAmp PowerFecal DNA Kit (Qiagen, Hilden, Germany), following the manufacturer's instructions. The concentration and purity of the genomic DNA were checked using a UV spectrophotometer (Mecasys, Daejeon, Korea). Amplification and sequencing of the V3-V4 hypervariable region of the 16S rRNA gene was performed using an Illumina MiSeq platform (Illumina, San Diego, CA, USA).

We performed alpha-diversity and taxonomic analyses of the raw paired-end sequences using EZBioCloud pipeline [19]. Then the samples were grouped into microbiome taxonomic profile sets for further analyses. Relative abundance cut-offs at the phylum, family, and genus levels were set to $0.1 \%$. Charts depicting the results from the alpha-diversity and taxonomic analyses were generated using GraphPad Prism software version 8.4.2 (GraphPad, San Diego, California, USA). Principal Coordinate Analyses (PCoAs) were performed using Quantitative Insights Into Microbial Ecology software (QIIME, v. 1.9.0) [20] to calculate the $\beta$-diversity based on the Bray-Curtis distance metric, as previously described [21].

\section{Bioinformatic analyses}

To illustrate the shared and unique microbiota found in each dietary group, a Venn diagram was created using an online tool (http://bioinformatics.psb.ugent.be/webtools/Venn/). To identify taxonomic markers for each group, linear discriminant analysis effect size (LEfSe) [22] analyses were performed using an online tool published by the Huttenhower Lab (https://huttenhower. sph.harvard.edu/galaxy/). To illustrate the correlation between the gut microbiota and fecal SCFAs and lactate contents, a heatmap was created using R (v.4.0.2) [23]. A co-occurrence network was illustrated using Cytoscape v.3.8 [24]. Predictions of the Kyoto encyclopedia of genes and genomes (KEGG) functional pathways (level 3) were performed using Phylogenetic Investigation of Communities by Reconstruction of Unobserved States (PICRUSt) software [25]. Differential KEGG pathways between groups were identified using STAMP v.2.1.3 [26].

\section{Statistical analyses}

GraphPad Prism software version 8.4.2 (GraphPad) was utilized for statistical analyses. Data normality was analyzed using Shapiro-Wilk test. Significant differences were calculated using Kruskal-Wallis $\mathrm{H}$ test or Mann-Whitney U test, when appropriate. Permutational multivariate analysis of variance (PERMANOVA) was used to determine significant differences in PCoAs. Spearman's rank correlation coefficients were calculated in $\mathrm{R}$ (v.4.0.2). For all statistical analyses, significance was determined at $p<0.05, p<0.01$, and $p<0.001$.

\section{RESULTS}

Growth performance and fecal concentrations of short-chain fatty acids and lactate The growth performance of the pigs after 6 weeks of feeding with MSP supplementation is summarized in Table 1 . We found no significant differences in the body weight and average 
Table 1. Effect of multispecies probiotics (MSP) on the growth performance of weaned pigs after 6 weeks

\begin{tabular}{lrrc}
\hline \multicolumn{1}{c}{ Items } & \multicolumn{1}{c}{ CON } & \multicolumn{1}{c}{ MSP } & p-values ${ }^{1)}$ \\
\hline Initial body weight (kg) & $23.61 \pm 0.28$ & $23.48 \pm 0.26$ & 0.74 \\
Final body weight (kg) & $48.66 \pm 1.28$ & $46.61 \pm 1.30$ & 0.20 \\
Average daily gain (ADG) (kg) & $0.60 \pm 0.03$ & $0.55 \pm 0.03$ & 0.27
\end{tabular}

Values are expressed as mean \pm SEM.

${ }^{11} \mathrm{~A} p$-values were calculated using Mann-Whitney $U$ test. Significant difference was considered at $(p<0.05]$.

CON, basal diet ( $\mathrm{n}=18)$; MSP, basal diet with multispecies probiotics containing Bacillus amyloliquefaciens G10, Limosilactobacillus brevis M10, Levilactobacillus reuteri RTR $(\mathrm{n}=20)$.

daily gain (ADG) between pigs in the MSP and CON groups $(p>0.05)$, indicating that MSP administration did not affect growth performance. On the other hand, the fecal concentrations of formate (1.4-fold; $p<0.05)$, acetate (1.8-fold; $p<0.05)$, and lactate (2.3-fold; $p<0.01)$ were significantly higher in the MSP group (Table 2), whereas propionate and butyrate were tended to increase by $12 \%$ and 14\%, respectively with MSP feeding, but not significantly so (Table 2). These results indicate that MSP induced the significant increase in the production of lactate, acetate and formate directly and/or indirectly.

\section{Gut microbial richness and diversity}

A total of 3,731,423 16S rRNA gene sequences were generated from the CON and MSP fecal samples. After removal of low-quality, nontargeted and chimeric amplicons, an average of 59,185 valid reads were generated. The sequencing depth was deemed sufficient for metagenomic analyses according to Good's coverage scores for both the CON and MSP groups (98.7\% and 99.4\%, respectively). The alpha diversity indices Chao1 $(p<0.001)$ and abundance-based coverage estimator (ACE, $p<0.01$ ) were significantly lower in the fecal samples of the MSP group (Figs. 1a and b). By contrast, the Simpson index was significantly higher in MSP samples ( $p<0.05)$ (Fig. 1c). Moreover, the PCoA plot constructed based on Bray-Curtis dissimilarity index revealed that the MSP fecal microbiota clustered separately from that of the CON group (Fig. 1d; PERMANOVA, $p<0.001)$. Our results strongly suggest that MSP supplementation altered the gut microbiota structure of the pigs.

\section{Gut microbiota composition}

At the phylum level, we observed five abundant phyla among the groups (abundance cut-off was set at 0.1\%; Fig. 2a; Supplementary Table S2). Firmicutes and Bacteroidetes were the most

Table 2. Effect of multispecies probiotics (MSP) on the fecal short-chain fatty acids (SCFAs) and lactate concentrations of weaned pigs

\begin{tabular}{lccc}
\hline \multicolumn{1}{c}{ Items } & CON & MSP & $p$-values ${ }^{1)}$ \\
\hline Formate $(\mu \mathrm{moL} / \mathrm{g}$ dry feces $)$ & $161.70 \pm 20.10$ & $231.20 \pm 17.32$ & 0.02 \\
Acetate $(\mu \mathrm{moL} / \mathrm{g}$ dry feces) & $168.10 \pm 44.92$ & $294.60 \pm 34.06$ & 0.04 \\
Propionate $(\mu \mathrm{moL} / \mathrm{g}$ dry feces $)$ & $66.53 \pm 8.64$ & $74.46 \pm 3.97$ & 0.41 \\
Butyrate $(\mu \mathrm{moL} / \mathrm{g}$ dry feces $)$ & $49.16 \pm 8.62$ & $55.97 \pm 13.26$ & 0.67 \\
Lactate $(\mu \mathrm{moL} / \mathrm{g}$ dry feces $)$ & $69.10 \pm 16.80$ & $160.80 \pm 20.11$ & 0.001 \\
\hline
\end{tabular}

Values are expressed as mean \pm SEM.

${ }^{1)} A$-values were calculated using Mann-Whitney $U$ test. Significant difference was considered at $(p<0.05)$.

CON, basal diet ( $n=18)$; MSP, basal diet with multispecies probiotics containing Bacillus amyloliquefaciens G10, Limosilactobacillus brevis M10, Levilactobacillus reuteri RTR $(n=20)$. 
(a)

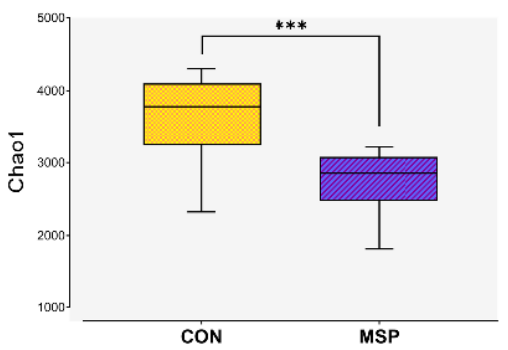

(d)

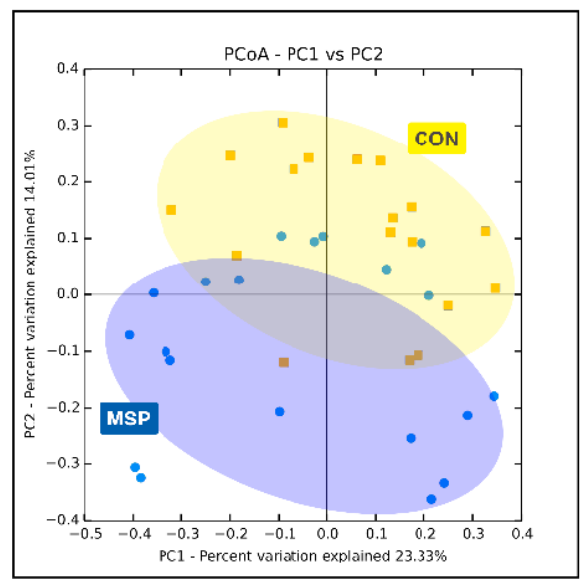

(b)

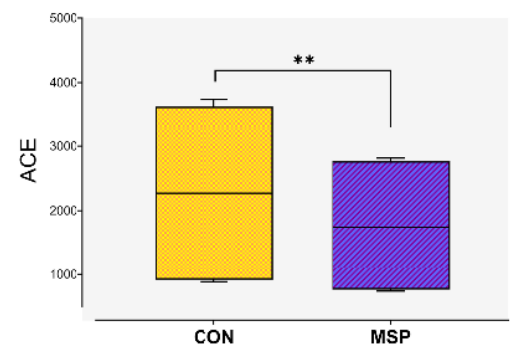

(c)

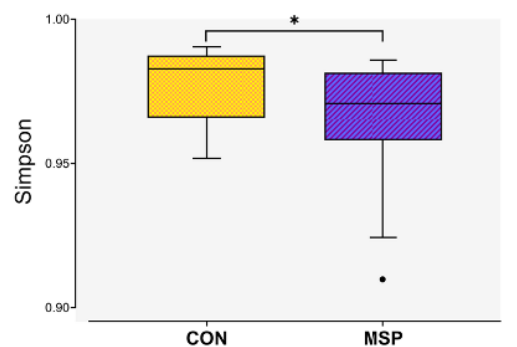

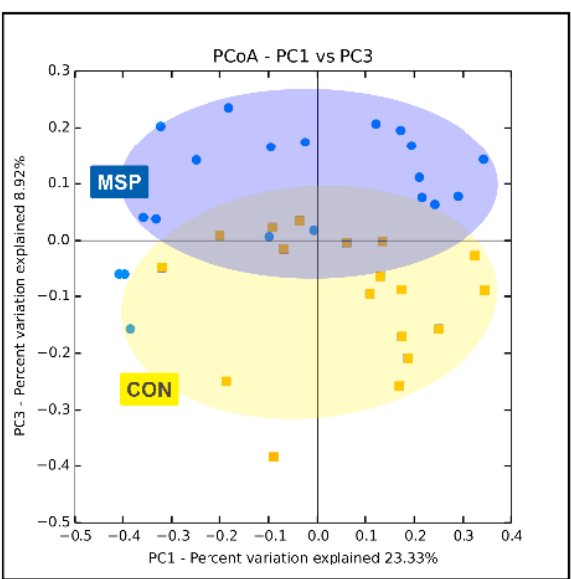

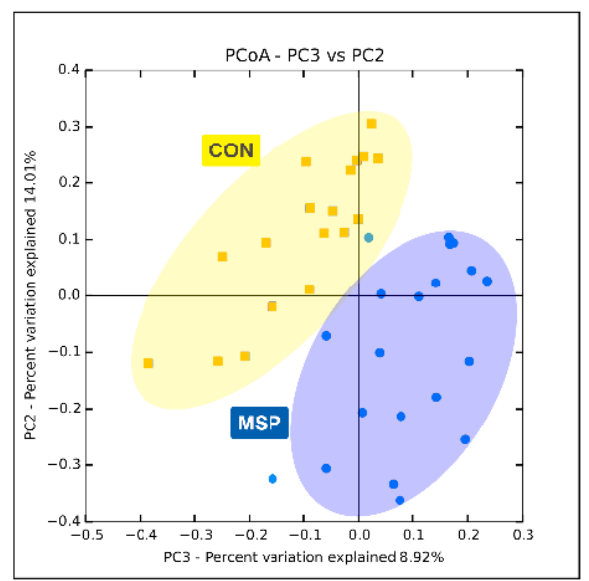

Fig. 1. Microbial richness and diversity. Comparison of alpha-diversity indices (a) Chao1, (b) ACE (abundance-based coverage estimator), (c) Simpson, and (d) clustering patterns of each group in PCoA plot. Significant values are denoted by ${ }^{*} p<0.05,{ }^{* *} p<0.01$ and ${ }^{* * *} p<0.001$. CON, basal diet ( $n=18$ ); MSP, basal diet with multispecies probiotics containing Bacillus amyloliquefaciens $\mathrm{G10}$, Levilactobacillus brevis M10, Limosilactobacillus reuteri RTR ( $\mathrm{n}=20$ ).

(a)



(b)


(c)

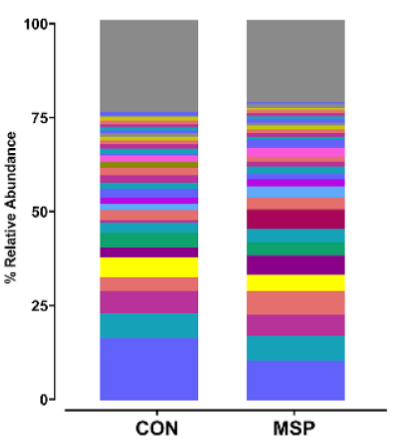

(d)

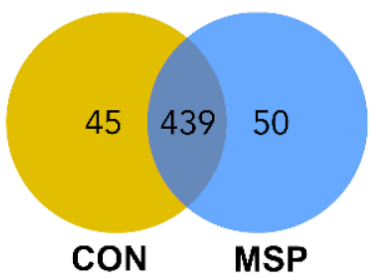

Fig. 2. Effect of multispecies probiotics (MSPs) on the gut microbiota composition of weaned pigs. Comparison of gut microbiota compositions between the diet groups at the (a) phylum, (b) family, and (c) genus levels. (d) Venn diagram showing the core microbiota of both groups and unique microbial communities within each group. Abundance cut-off was set to $>0.1 \%$. CON, basal diet $(n=18)$; MSP, basal diet with multispecies probiotics containing Bacillus amyloliquefaciens $\mathrm{G} 10$, Levilactobacillus brevis M10, Limosilactobacillus reuteri RTR ( $\mathrm{n}=20)$. 
abundant phyla across the groups, covering about $90 \%$ of the total sequence reads. We found that MSP supplementation significantly increased the abundance of Firmicutes $(59.07 \%$ vs. $65.67 \%$ for CON vs. MSP, respectively; $p<0.01)$, and decreased the abundance of Bacteroidetes $(34.27 \%$ vs. $24.41 \%$ for CON vs. MSP, respectively; $p<0.01)$. Consequently, the Firmicutes:Bacteroidetes $(\mathrm{F}: \mathrm{B})$ ratio was significantly higher in the MSP group compared to in the CON group (1.72 vs. 2.69 for $\mathrm{CON}$ and MSP, respectively; $p<0.001$ ). Moreover, the abundance of the phylum Actinobacteria was remarkably enriched following MSP supplementation (0.90\% vs 5.61\%; $p<0.01)$. By contrast, the abundance of the phylum Chlamydiae was greatly reduced after MSP supplementation (0.19\% vs. $0.0003 \% ; p<0.05$ ). At the family level, Ruminococcaceae, Prevotellaceae and Lachnospiraceae were the most abundant bacterial families (Fig. 2b; Supplementary Table S3). The intake of MSP lowered the abundance of Prevotellaceae (19.80\% vs. $12.84 \% ; p<0.01)$.

At the genus level, we identified a total of 534 genera across the groups (Fig. 2d), of which 439 were shared between the groups, while 45 and 50 were unique to the CON and MSP groups, respectively. Fig. 2c and Supplementary Table S4 summarizes the distribution of the genera between the groups. Prevotella was the most abundant genus in both groups. Supplementation with MSP led to the significant enrichment of Olsenella (0.06\% vs. 5.20\%; $p<0.01)$, Catonella (0.32\% vs. $2.20 \% ; p<0.05)$, Acidaminococcus (0.29\% vs. $1.19 \% ; p<0.01)$, Dialister (3.51\% vs. 6.14\%), Christensenellaceae PAC001115 (2.77\% vs. 5.00\%), Ruminococcaceae PAC000661 (1.58\% vs. $2.81 \% ; p<0.05)$, and Catenibacterium (0.21\% vs. $0.45 \% ; p<0.05)$. In addition, the abundance of Lactobacillus was also slightly higher (1.83\% vs. 2.13\%) in the MSP group. On the other hand, populations of Prevotella (16.40\% vs. $10.45 \%$; $p<0.01)$, Roseburia $(1.51 \%$ vs. $0.67 \%$; $p<0.05)$, Alloprevotella (1.03\% vs. $0.52 \%$; $p<0.01$, Escherichia (0.32\% vs. $0.05 \%)$, and Chlamydia (0.19\% vs. $0.0003 \% ; p<0.05)$ were significantly lower in MSP-fed pigs.

Next, we identified potential taxonomic markers in the gut microbiota of the pigs using LEfSe (Fig. 3). The enriched abundances of Olsenella, Catonella, Ruminococcaceae PAC000661,

(a)

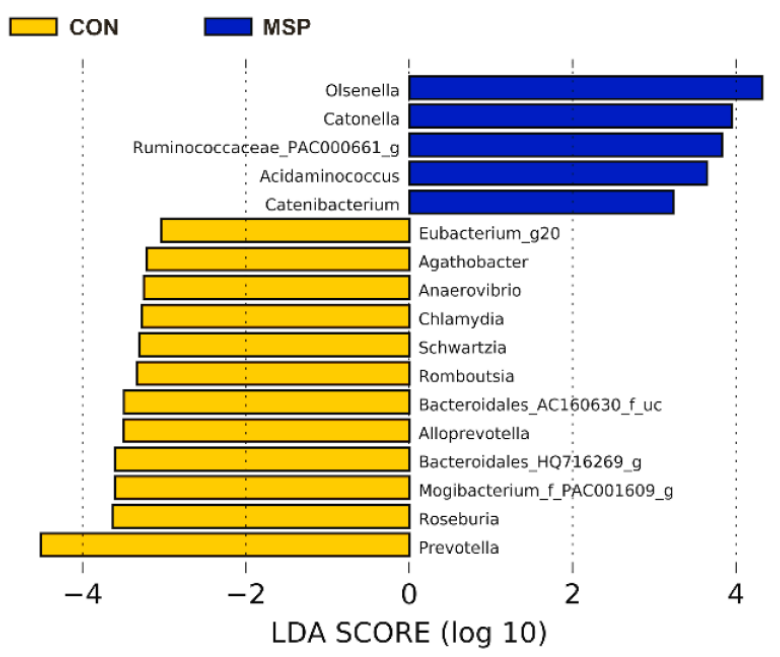

(b)



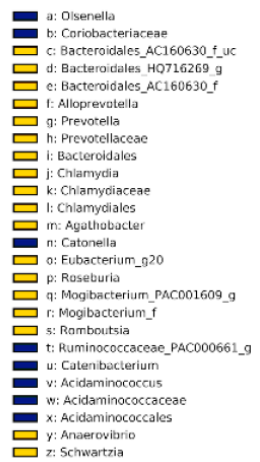

Fig. 3. Taxonomic feature identification between the CON (control) and multispecies probiotic (MSP) groups using linear discriminant analysis effect size (LEfSe) analyses. (a) Histogram showing the taxonomical features identified between the CON and MSP groups, and (b) cladogram showing the phylogenetic relationships among taxonomical features identified between the CON and MSP groups. Significance was present at $p<0.05$ for the LEfSe analyses for both Kruskal- Wallis and Wilcoxon tests, and the minimum linear discriminant analysis (LDA) score was set to 2.0. 
Acidaminococcus, and Catenibacterium were identified as taxonomic markers for the MSP group $(p<0.05)$. By contrast, Prevotella, members of the order Bacteroidales (AC160630, HQ716269), Mogibacterium PAC001609, Romboutsia, Anaerovibrio, Agathobacther, Alloprevotella, Roseburia, Schwartzia, and Eubacterium_g20 were identified as taxonomic features of the CON group $(p<$ 0.05). Plots depicting the abundances of the taxonomic markers are shown in Fig. 4. Our results further suggest that MSP supplementation elicits a modulatory effect on the gut microbiota of pigs.

\section{Correlation between gut microbiota and SCFAs and lactate concentration}

To identify the associations between the gut microbiota and the fecal SCFAs and lactate concentrations, we conducted a correlation analysis by calculating the Spearman's correlation coefficients (Fig. 5a). It showed that Olsenella, Catenibacterium, and Ruminococcaceae PAC000661 have significantly correlated with lactate $(p<0.05)$. Olsenella and Lactobacillus were observed to be significantly associated with acetate $(p<0.05)$, while Acidaminococcus was significantly correlated with formate $(p<0.05)$. By contrast, Prevotella $(p<0.05)$, Mogibacterium $(p<0.01)$, and Alloprevotella $(p<0.05)$ had significant negative correlations with lactate. In addition, Prevotella and Chlamydia were observed to be negatively linked with acetate $(p<0.05)$, while Alloprevotella was negatively associated with formate $(p<0.05)$. On the other hand, order Bacteroidales HQ716269 $(p<0.05)$, Roseburia, Agathobacter, Alloprevotella, Prevotella, and Anaerovibrio were positively linked with butyrate. These observed associations were supported by the network map (Fig. 5b), showing positive correlation among MSP-enriched taxa, lactate, formate, and acetate; Catonella has positive relationship with butyrate. Meanwhile, MSP-diminished taxa co-occurred with each other; order Bacteroidales HQ716269 positively correlated with butyrate. These observations suggest the potential interplay between the MSP-modulated gut microbiota and the production of SCFA and lactate.

\section{Predicted Kyoto encyclopedia of genes and genomes functional pathways in the gut microbiome}

To predict functional markers in the gut microbiome of the pigs, we performed PICRUSt analyses [25]. A total of $267 \mathrm{KEGG}$ pathways were predicted across both groups (Supplementary Table S5). We found that 30 of the predicted KEGG pathways were significantly altered in the MSP group (Fig. 6). KEGG pathways including transporters, pentose phosphate pathway, amino acid metabolism, glycerolipid metabolism, and fatty acid metabolism were significantly enriched in the MSP group compared to controls $(p<0.05)$. Other KEGG pathways, such as starch and sucrose metabolism, cyanoamino acid metabolism, nitrogen metabolism, alanine, aspartate and glutamate metabolism, phenylpropanoid biosynthesis, chaperones and folding catalysts, and pantothenate and CoA biosynthesis were enriched in the CON group $(p<0.05)$. This indicates that MSP supplementation altered the functional capacity of the pig microbiota.

\section{DISCUSSION}

We investigated the effects of supplementation with MSP containing B. amyloliquefaciens G10, $L$. reuteri $\mathrm{RTR}$, and $L$. brevis $\mathrm{M} 10$ on the growth performance and fecal SCFA levels in weaned pigs. Our in vitro studies showed no antagonistic effect among these strains, but showed synergistic effect on the diversity of fecal microbiota in pigs (data not shown). Some previous studies have suggested that MSP supplementation in pigs leads to improved growth performance [13,14]. However, in the present study, no significant differences in body weight or ADG were observed in MSP-fed pigs. This observation is similar to those of other studies, in which no observable increase 
(a)


Ruminococcaceae PAC000661

(b)


Fig. 4. Relative abundances of selected taxa. Comparisons of the relative abundances of selected taxa identified by the linear discriminant analysis effect size (LEfSe) analyses. A p-values were calculated using Kruskal-Wallis $\mathrm{H}$ test. Significant values are denoted by ${ }^{*} p<0.05,{ }^{* *} p<0.01$ and ${ }^{* * *} p<0.001$. CON, basal diet $(n=18)$; MSP, basal diet with multispecies probiotics containing Bacillus amyloliquefaciens $\mathrm{G} 10$, Levilactobacillus brevis M10, Limosilactobacillus reuteri $\operatorname{RTR}(\mathrm{n}=20)$. 
a)



b)

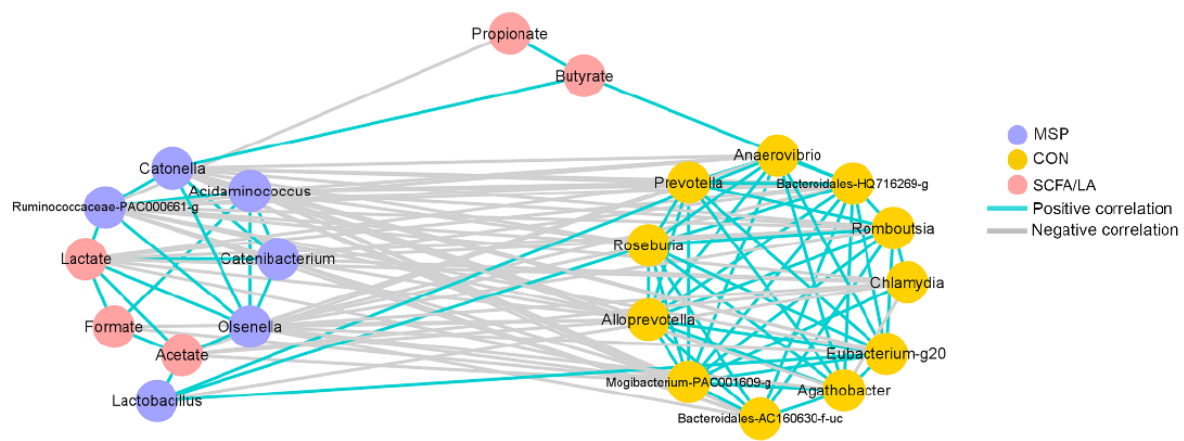

Fig. 5. Correlation between the gut microbiota and short-chain fatty acids (SCFA) and lactate (LA). (a) Heatmap and (b) co-occurrence network showing associations between MSP-modulated taxa and fecal SCFA's and lactate. The heatmap was constructed according to Spearman's correlation. The degree of correlation is represented by color hue: orange represents positive correlation; blue represents negative correlation. Significant values are denoted as ${ }^{*} p<0.05$. CON, basal diet $(n=18)$; MSP, basal diet with multispecies probiotics containing Bacillus amyloliquefaciens $\mathrm{G} 10$, Levilactobacillus brevis M10, Limosilactobacillus reuteri RTR ( $\mathrm{n}=20$ ).

on body weight or ADG in pigs treated with probiotics was found [27-30]. This phenomenon may be due to multiple factors influencing body weight gain such as feeding concentration and period, environmental stress, animal age, or the specific characteristics of the probiotic strains [29,30].

Multiple studies have revealed the role of SCFAs in metabolic homeostasis and overall gut health in humans and animals. SCFAs (mainly acetate, butyrate, and propionate) are energy source for enterocytes, and known to improve gut integrity, regulate glucose and lipid homeostasis, and improve immune function [1,2,31]. Production of SCFAs and lactate by the gut microbiota has been extensively described in other studies [5,31]. The modulating effect of SCFAs on the host's health is mainly attributed to its involvement in the inhibition of histone deacetylases and activation of G-protein-coupled receptors, such as GPR41, GPR43, and GPR109A [5,32]. Host diets play a crucial role in determining the level of SCFAs produced by gut microbiota, such that, diets rich in fibers or supplemented by prebiotics can trigger the production of high levels of SCFAs $[7,9,10]$. In addition, probiotics have demonstrated the ability to affect the production of SCFAs [11,12]. In the present study, we investigated the effect of MSP supplementation on the SCFAs and lactate levels in pigs. After the feeding period, we found a significant increase in the fecal levels of formate, acetate, and lactate in MSP-fed pigs. Similarly, Nagata et al. [33] and Alcon-Giner et al. [34] reported increases in acetate level following probiotic treatment. Acetate improves metabolic homeostasis, energy utilization, and appetite regulation $[35,36]$. The role of formate in gut health is not yet fully understood, although it can impart protective properties against pathogens such as Escherichia coli and Salmonella [37,38]. Lactate, which is mainly produced by lactic acid bacteria and 


\begin{tabular}{|c|c|c|c|c|}
\hline \multirow{2}{*}{\multicolumn{2}{|c|}{ Transporters }} & \multicolumn{3}{|c|}{$95 \%$ confidence intervals } \\
\hline & & & 0 & 0.043 \\
\hline Starch and sucrose metabolism & & & ○ & 0.011 \\
\hline Cyanoamino acid metabolism $\exists$ & & & 0 & $2.00 \mathrm{e}-3$ \\
\hline Nitrogen metabolism $\boxminus$ & & & 0 & $2.00 \mathrm{e}-3$ \\
\hline "Alanine, aspartate and glutamate metabolism" & & & b & 0.019 \\
\hline Phenylpropanoid biosynthesis & & & b & $2.00 \mathrm{e}-3$ \\
\hline Chaperones and folding catalysts $\Xi$ & & & 0 & 0.036 \\
\hline Pantothenate and CoA biosynthesis $日$ & & & Q & $4.00 \mathrm{e}-3$ \\
\hline Benzoate degradation $\}$ & & & 0 & $9.99 \mathrm{e}-4$ \\
\hline "Phenylalanine, tyrosine and tryptophan biosynthesis" & & & 0 & 0.030 \\
\hline Ubiquinone and other terpenoid-quinone biosynthesis & & & $\delta$ & 0.019 \\
\hline Sulfur metabolism $\mathrm{B}$ & & & 0 & $4.00 \mathrm{e}-3$ \\
\hline Pentose phosphate pathway $\exists$ & & & 0 & 0.014 \\
\hline Pentose and glucuronate interconversions $日$ & & & Q & 0.044 \\
\hline Histidine metabolism 日 & & & 0 & 0.023 \\
\hline Translation proteins $\Xi$ & & & 0 & 0.035 \\
\hline Amino acid metabolism & & & o & 0.032 \\
\hline Glycerolipid metabolism $\exists$ & & & b & 0.047 \\
\hline "Valine, leucine and isoleucine degradation" & & & 0 & 0.015 \\
\hline Fatty acid metabolism & & & Q & 0.016 \\
\hline Glycerophospholipid metabolism 日 & & & O & 0.049 \\
\hline Other transporters $\mathrm{G}$ & & & O & 0.011 \\
\hline Lysine degradation & & & b & $3.00 \mathrm{e}-3$ \\
\hline RNA polymerase ] & & & d & 0.012 \\
\hline Cellular antigens | & & & Q & 0.028 \\
\hline Limonene and pinene degradation | & & & Q & 0.021 \\
\hline Protein digestion and absorption | & & & 0 & 0.014 \\
\hline D-Alanine metabolism $\mathbf{1}$ & & & 0 & $2.00 \mathrm{e}-3$ \\
\hline Electron transfer carriers | & & & o & $4.00 \mathrm{e}-3$ \\
\hline Phosphonate and phosphinate metabolism | & & & 0 & 0.036 \\
\hline Bisphenol degradation | & & & Q & 0.019 \\
\hline Drug metabolism - cytochrome P450| & & & 0 & 0.031 \\
\hline Atrazine degradation | & & & 0 & 0.011 \\
\hline Metabolism of xenobiotics by cytochrome P450 | & & & 0 & 0.031 \\
\hline GnRH signaling pathway | & & & $\dot{\varphi}$ & $4.00 \mathrm{e}-3$ \\
\hline Fc gamma R-mediated phagocytosis | & & & 0 & $4.00 \mathrm{e}-3$ \\
\hline Glycosphingolipid biosynthesis - lacto and neolact... | & & & 0 & $6.39 \mathrm{e}-3$ \\
\hline & $\perp$ & $1 \quad 1$ & $\perp+1+1+1$ & \\
\hline 0.0 & 6.5 & $15-10-5$ & $\begin{array}{llllll}0 & 5 & 10 & 15 & 20 & 25\end{array}$ & 5 \\
\hline
\end{tabular}

Fig. 6. Kyoto encyclopedia of genes and genomes (KEGG) functional pathways predicted in the gut microbiomes of the control (CON) and multispecies probiotic (MSP) groups via phylogenetic investigation of communities by reconstruction of unobserved states (PICRUSt) analyses. The plot shows the KEGG pathways that were significantly altered by supplementation with MSPs containing Bacillus amyloliquefaciens $\mathrm{G} 10$, Levilactobacillus brevis $\mathrm{M} 10$ and Limosilactobacillus reuteri RTR.

bifidobacteria, serves as an intermediate molecule for acetate, butyrate, and propionate production by lactate-utilizing bacteria $[4,39]$. Although the fecal levels of butyrate and propionate were slightly higher in MSP-fed pigs, no significant differences were found in the present study, as observed previously in other studies $[27,28]$.

At the phylum level, we observed elevated populations of Firmicutes in MSP-fed pigs compared to in the control group. A positive correlation between the abundance of Firmicutes and acetate and lactate levels has previously been reported [40,41]. Moreover, we found that the phylum Actinobacteria was also enriched following MSP supplementation. By contrast, MSP supplementation drastically reduced the population of the phylum Chlamydiae, which is responsible for various diseases in pigs [42].

At the genus level, we found that MSP supplementation caused the significant enrichment of Olsenella, Catonella, Acidaminococcus, Catenibacterium, and Ruminococcaceae PAC000661; these 
genera formed positive relationships with increased levels of acetate, formate, and lactate. Increases in the abundance of Olsenella have been observed in several studies that have examined the effects of supplementing diets with probiotics, complex carbohydrates, or prebiotics [43,44]. Olsenella is a carbohydrate fermenter and producer of acetate and lactate [45,46], which is consistent with our correlation results. Likewise, the genus Catenibacterium is positively associated with high SCFA levels [47]. Acidaminococcus can convert glutamate into acetate and butyrate [48] and Catonella enrichment is correlated with reduced diarrheal severity and an increase in acetate and butyrate levels in patients with irritable bowel syndrome treated with synbiotics [49]; this suggests that the prevalence of Acidaminococcus and Catonella improved the fecal level of acetate in MSP-fed pigs. In addition, the population of Lactobacillus was observed to increase upon MSP-feeding, and has shown to have positive correlation with acetate and lactate. Relative abundances of Bifidobacterium and other lactic acid bacteria was below cut-off $(0.1 \%)$ for this study. The increase in Lactobacillus may be due to the supplementation of probiotics, which is similar to previous study [50]. Finally, Ruminococcaceae produces SCFAs from complex plant materials [51]. Elevated populations of Ruminococcaceae are negatively associated with constipation, inflammation, and liver diseases, and are typically observed in healthy subjects [52,53]. Our results indicate the potential positive effects of dietary MSP inclusion on pig health, especially on SCFA production, which should be investigated further.

MSP supplementation also decreased the abundance of Prevotella, Roseburia, Alloprevotella, Romboutsia, Anaerovibrio, Mogibacterium, the order Bacteroidales, Agathobacter, and Eubacterium, as revealed by LEfSe analyses. Furthermore, we found positive associations between order Bacteroidales, Prevotella, Roseburia, Agathobacter, and Alloprevotela, and butyrate levels. Our results are consistent with the fact that several members of the order Bacteroidales produce butyrate [54]. Prevotella has an increased prevalence in subjects with plant-rich diets and produces SCFAs [55,56]. Likewise, Alloprevotella and Anaerovibrio produce SCFAs [57-59]. However, elevated abundance of Prevotella are associated with inflammation, hypertension, insulin resistance, and liver disease [60,61]. In addition, Alloprevotella and Anerovibrio are present in individuals with colitis and chronic kidney disease [62,63], and rats with type 2 diabetes [64], respectively. Roseburia and Agathobacter are beneficial gut bacteria that produce SCFAs, particularly butyrate $[65,66]$. Our findings support these studies in that the abundance of these genera positively correlated with butyrate. Our results suggest that the decrease in the abundances of Prevotella, Roseburia, Agathobacter, the order Bacteroidales, Alloprevotela, and Anaerovibrio may have lowered the butyrate levels in the MSP-fed group due to a decrease in metabolite cross-feeding from acetate or lactate into butyrate $[67,68]$. Further studies are needed to investigate the possible interplay between probiotics and the fermentation capacity of these genera.

Interestingly, we observed a dramatic decline in the abundance of Chlamydia and Escherichia after supplementation with MSPs. Furthermore, we found that Chlamydia negatively correlated with acetate and lactate. Both Chlamydia and Escherichia are recognized pathogens in both humans and pigs [42]. SCFAs impart protective effects against enteric pathogens, either by inducing $\mathrm{pH}$ stress or by promoting inflammasomes $[69,70]$. Our results provide further evidence for enhanced SCFA production by MSP supplementation and their beneficial effects of gut health.

Finally, our PICRUSt analyses revealed that MSP supplementation also altered the metabolic functions of the gut microbiome. MSP treatment enriched several KEGG pathways including transporters, pentose phosphate pathway, amino acid metabolism, glycerolipid metabolism, and fatty acid metabolism. The elevated abundances of Firmicutes, as well as Lachnospiraceae and Ruminococcaceae may explain the abundance of transporters in MSP-fed pigs [5,51]. Moreover, the gut microbiota (mainly Firmicutes) utilizes the pentose phosphate pathway to degrade 
complex fibers, thereby producing SCFAs [71,72]. Amino acid metabolism by gut microbiota may also provide substrates for SCFA production [73]. Probiotic supplementation can increase the abundance of glycerolipid metabolism and fatty acid metabolism pathways, which are associated with improved host metabolic health [74,75]. These MSP-mediated changes in functional pathways further support our findings that MSP supplementation can modulate the gut microbiota and SCFA levels in pigs.

In conclusion, we found that MSP supplementation resulted in significantly higher levels of fecal acetate, formate, and lactate in pigs. Moreover, MSP supplementation altered the gut microbiota of the pigs by increasing the population of potentially beneficial bacteria such as Olsenella, Catonella, Catenibacterium, Acidaminococcus, and Ruminococcaceae. Dietary MSP also caused a decline in the abundance of pathogenic bacteria such as Escherichia and Chlamydia. The modulation of the gut microbiota was observed to be strongly correlated with the changes in fecal SCFAs and lactate levels, which suggests an interplay between the two. Furthermore, we found changes in the functional pathways present within the gut microbiome. Our results demonstrate the potential use of MSPs for improving the gut health of animals and humans. Further in-depth investigations are needed to explore the interplay between the taxa identified in this study and SCFAs and its overall effect on host metabolic health.

\section{SUPPLEMENTARY MATERIALS}

Supplementary materials are only available online from: https://doi.org/10.5187/jast.2021.e94.

\section{REFERENCES}

1. Morrison DJ, Preston T. Formation of short chain fatty acids by the gut microbiota and their impact on human metabolism. Gut Microbes. 2016;7:189-200. https://doi.org/10.1080/19490 976.2015.1134082

2. Granado-Serrano AB, Martín-Garí M, Sánchez V, Riart Solans M, Berdún R, Ludwig IA, et al. Faecal bacterial and short-chain fatty acids signature in hypercholesterolemia. Sci Rep. 2019;9:1772.https://doi.org/10.1038/s41598-019-38874-3

3. Macfarlane S, Macfarlane GT. Regulation of short-chain fatty acid production. Proc Nutr Soc. 2003;62:67-72. https://doi.org/10.1079/PNS2002207

4. Louis P, Flint HJ. Formation of propionate and butyrate by the human colonic microbiota. Environ Microbiol. 2017;19:29-41.https://doi.org/10.1111/1462-2920.13589

5. Tan J, McKenzie C, Potamitis M, Thorburn AN, Mackay CR, Macia L. The role of shortchain fatty acids in health and disease. In: Alt FW, editor. Advances in immunology. Amsterdam: Academic Press; 2014. p. 91-119.

6. Belenguer A, Duncan SH, Calder AG, Holtrop G, Louis P, Lobley GE, et al. Two routes of metabolic cross-feeding between Bifidobacterium adolescentis and butyrate-producing anaerobes from the human gut. Appl Environ Microbiol. 2006;72:3593-9. https://doi.org/10.1128/ AEM.72.5.3593-3599.2006

7. De Filippis F, Pellegrini N, Vannini L, Jeffery IB, La Storia A, Laghi L, et al. High-level adherence to a Mediterranean diet beneficially impacts the gut microbiota and associated metabolome. Gut. 2016;65:1812-21. https://doi.org/10.1136/gutjnl-2015-309957

8. Krznarić Ž, Vranešić Bender D, Meštrović T. The Mediterranean diet and its association with selected gut bacteria. Curr Opin Clin Nutr Metab Care. 2019;22:401-6. https://doi. org/10.1097/MCO.0000000000000587 
9. Hedemann MS, Bach Knudsen KE. Resistant starch for weaning pigs: effect on concentration of short chain fatty acids in digesta and intestinal morphology. Livest Sci. 2007;108:175-7. https://doi.org/10.1016/j.livsci.2007.01.045

10. Jha R, Leterme P. Feed ingredients differing in fermentable fibre and indigestible protein content affect fermentation metabolites and faecal nitrogen excretion in growing pigs. Animal. 2012;6:603-11. https://doi.org/10.1017/S1751731111001844

11. Wang Y, Dilidaxi D, Wu Y, Sailike J, Sun X, Nabi X. Composite probiotics alleviate type 2 diabetes by regulating intestinal microbiota and inducing GLP-1 secretion in $\mathrm{db} / \mathrm{db}$ mice. Biomed Pharmacother. 2020;125:109914. https://doi.org/10.1016/j.biopha.2020.109914

12. Joseph N, Vasodavan K, Saipudin NA, Yusof BNM, Kumar S, Nordin SA. Gut microbiota and short-chain fatty acids (SCFAs) profiles of normal and overweight school children in Selangor after probiotics administration. J Funct Foods. 2019;57:103-11. https://doi.org/10.1016/ j.jff.2019.03.042

13. Lu X, Zhang M, Zhao L, Ge K, Wang Z, Jun L, et al. Growth performance and post-weaning diarrhea in piglets fed a diet supplemented with probiotic complexes. J Microbiol Biotechnol. 2018;28:1791-9. https://doi.org/10.4014/jmb.1807.07026

14. Balasubramanian B, Lee SI, Kim IH. Inclusion of dietary multi-species probiotic on growth performance, nutrient digestibility, meat quality traits, faecal microbiota and diarrhoea score in growing-finishing pigs. Ital J Anim Sci. 2018;17:100-6. https://doi.org/10.1080/182805 1X.2017.1340097

15. Neveling DP, van Emmenes L, Ahire JJ, Pieterse E, Smith C, Dicks LMT. Effect of a multi-species probiotic on the colonisation of Salmonella in broilers. Probiotics Antimicrob Proteins. 2020;12:896-905. https://doi.org/10.1007/s12602-019-09593-y

16. Wang K, Chen G, Cao G, Xu Y, Wang Y, Yang C. Effects of Clostridium butyricum and Enterococcus faecalis on growth performance, intestinal structure, and inflammation in lipopolysaccharide-challenged weaned piglets. J Anim Sci. 2019;97:4140-51. https://doi.org/10.1093/ jas/skz235

17. Deda O, Chatziioannou AC, Fasoula S, Palachanis D, Raikos N, Theodoridis GA, et al. Sample preparation optimization in fecal metabolic profiling. J Chromatogr B. 2017;1047:115-23. https://doi.org/10.1016/j.jchromb.2016.06.047

18. De Baere S, Eeckhaut V, Steppe M, De Maesschalck C, De Backer P, Van Immerseel F, et al. Development of a HPLC-UV method for the quantitative determination of four short-chain fatty acids and lactic acid produced by intestinal bacteria during in vitro fermentation. J Pharm Biomed Anal. 2013;80:107-15. https://doi.org/10.1016/j.jpba.2013.02.032

19. Yoon SH, Ha SM, Kwon S, Lim J, Kim Y, Seo H, et al. Introducing EzBioCloud: a taxonomically united database of $16 \mathrm{~S}$ rRNA gene sequences and whole-genome assemblies. Int J Syst Evol Microbiol. 2017;67:1613-7. https://doi.org/10.1099/ijsem.0.001755

20. Caporaso JG, Kuczynski J, Stombaugh J, Bittinger K, Bushman FD, Costello EK, et al. QIIME allows analysis of high-throughput community sequencing data. Nat Methods. 2010;7:335-6. https://doi.org/10.1038/nmeth.f.303

21. Oh JK, Amoranto MBC, Oh NS, Kim S, Lee JY, Oh YN, et al. Synergistic effect of Lactobacillus gasseri and Cudrania tricuspidata on the modulation of body weight and gut microbiota structure in diet-induced obese mice. Appl Microbiol Biotechnol. 2020;104:6273-85. https:// doi.org/10.1007/s00253-020-10634-8

22. Segata N, Izard J, Waldron L, Gevers D, Miropolsky L, Garrett WS, et al. Metagenomic biomarker discovery and explanation. Genome Biol. 2011;12:R60. https://doi.org/10.1186/gb2011-12-6-r60 
23. R Core Team. R: a language and environment for statistical computing. Vienna, Austria: $\mathrm{R}$ Foundation for Statistical Computing; 2020.

24. Shannon P, Markiel A, Ozier O, Baliga NS, Wang JT, Ramage D, et al. Cytoscape: a software environment for integrated models of biomolecular interaction networks. Genome Res. 2003;13:2498-504. https://doi.org/10.1101/gr.1239303

25. Langille MGI, Zaneveld J, Caporaso JG, McDonald D, Knights D, Reyes JA, et al. Predictive functional profiling of microbial communities using $16 \mathrm{~S}$ rRNA marker gene sequences. Nat Biotechnol. 2013;31:814-21. https://doi.org/10.1038/nbt.2676

26. Parks DH, Tyson GW, Hugenholtz P, Beiko RG. STAMP: statistical analysis of taxonomic and functional profiles. Bioinformatics. 2014;30:3123-4. https://doi.org/10.1093/bioinformatics/btu494

27. Huang C, Qiao S, Li D, Piao X, Ren J. Effects of Lactobacilli on the performance, diarrhea incidence, VFA concentration and gastrointestinal microbial flora of weaning pigs. Asian-Australas J Anim Sci. 2004;17:401-9. https://doi.org/10.5713/ajas.2004.401

28. Mair C, Plitzner C, Domig KJ, Schedle K, Windisch W. Impact of inulin and a multispecies probiotic formulation on performance, microbial ecology and concomitant fermentation patterns in newly weaned piglets. J Anim Physiol Anim Nutr. 2010;94:e164-77. https://doi. org/10.1111/j.1439-0396.2010.01000.x

29. Lähteinen T, Rinttilä T, Koort JMK, Kant R, Levonen K, Jakava-Viljanen M, et al. Effect of a multispecies Lactobacillus formulation as a feeding supplement on the performance and immune function of piglets. Livest Sci. 2015;180:164-71. https://doi.org/10.1016/j.livsci.2015.07.016

30. Jaworski NW, Owusu-Asiedu A, Walsh MC, McCann JC, Loor JJ, Stein HH. Effects of a 3 strain Bacillus-based direct-fed microbial and dietary fiber concentration on growth performance and expression of genes related to absorption and metabolism of volatile fatty acids in weanling pigs.J Anim Sci. 2017;95:308-19. https://doi.org/10.2527/jas.2016.0557

31. Markowiak-Kopeć P, Śliżewska K. The effect of probiotics on the production of short-chain fatty acids by human intestinal microbiome. Nutrients. 2020;12:1107. https://doi.org/10.3390/ nu12041107

32. Śliżewska K, Markowiak-Kopeć P, Żbikowski A, Szeleszczuk P. The effect of synbiotic preparations on the intestinal microbiota and her metabolism in broiler chickens. Sci Rep. 2020;10:4281. https://doi.org/10.1038/s41598-020-61256-z

33. Nagata S, Chiba Y, Wang C, Yamashiro Y. The effects of the Lactobacillus casei strain on obesity in children: a pilot study. Benefic Microbes. 2017;8:535-43. https://doi.org/10.3920/ BM2016.0170

34. Alcon-Giner C, Dalby MJ, Caim S, Ketskemety J, Shaw A, Sim K, et al. Microbiota supplementation with Bifidobacterium and Lactobacillus modifies the preterm infant gut microbiota and metabolome: an observational study. Cell Rep Med. 2020;1:100077. https://doi. org/10.1016/j.xcrm.2020.100077

35. Shimizu H, Masujima Y, Ushiroda C, Mizushima R, Taira S, Ohue-Kitano R, et al. Dietary short-chain fatty acid intake improves the hepatic metabolic condition via FFAR3. Sci Rep. 2019;9:16574. https://doi.org/10.1038/S41598-019-53242-x

36. Canfora EE, Jocken JW, Blaak EE. Short-chain fatty acids in control of body weight and insulin sensitivity. Nat Rev Endocrinol. 2015;11:577-91. https://doi.org/10.1038/nrendo.2015.128

37. Gómez-García M, Sol C, de Nova PJG, Puyalto M, Mesas L, Puente H, et al. Antimicrobial activity of a selection of organic acids, their salts and essential oils against swine enteropathogenic bacteria. Porcine Health Manag. 2019;5:32. https://doi.org/10.1186/s40813-019-0139-4 
38. Creus E, Pérez JF, Peralta B, Baucells F, Mateu E. Effect of acidified feed on the prevalence of Salmonella in market-age pigs. Zoonoses Public Health. 2007;54:314-9. https://doi. org/10.1111/j.1863-2378.2007.01069.x

39. Ríos-Covián D, Ruas-Madiedo P, Margolles A, Gueimonde M, de Los Reyes-Gavilán CG, Salazar N. Intestinal short chain fatty acids and their link with diet and human health. Front Microbiol. 2016;7:185.https://doi.org/10.3389/fmicb.2016.00185

40. Chevalier C, Stojanović O, Colin DJ, Suarez-Zamorano N, Tarallo V, Veyrat-Durebex C, et al. Gut microbiota orchestrates energy homeostasis during cold. Cell. 2015;163:1360-74. https:// doi.org/10.1016/j.cell.2015.11.004

41. Li G, Xie C, Lu S, Nichols RG, Tian Y, Li L, et al. Intermittent fasting promotes white adipose browning and decreases obesity by shaping the gut microbiota. Cell Metab. 2017;26:67285.https://doi.org/10.1016/j.cmet.2017.08.019

42. Schautteet K, Vanrompay D. Chlamydiaceae infections in pig. Vet Res. 2011;42:29. https://doi. org/10.1186/1297-9716-42-29

43. Chen L, Zhang L, Wang W, Qiu W, Liu L, Ning A, et al. Polysaccharides isolated from Cordyceps sinensis contribute to the progression of NASH by modifying the gut microbiota in mice fed a high-fat diet. PLOS ONE. 2020;15:e0232972. https://doi.org/10.1371/journal. pone. 0232972

44. Zhong H, Abdullah, Deng L, Zhao M, Tang J, Liu T, et al. Probiotic-fermented blueberry juice prevents obesity and hyperglycemia in high fat diet-fed mice in association with modulating the gut microbiota. Food Funct. 2020;11:9192-207. https://doi.org/10.1039/D0FO00334D

45. Kraatz M, Wallace RJ, Svensson L. Olsenella umbonata sp. nov., a microaerotolerant anaerobic lactic acid bacterium from the sheep rumen and pig jejunum, and emended descriptions of Olsenella, Olsenella uli and Olsenella profusa. Int J Syst Evol Microbiol. 2011;61:795-803. https://doi.org/10.1099/ijs.0.022954-0

46. Li W, Zhang K, Yang H. Pectin alleviates high fat (lard) diet-induced nonalcoholic fatty liver disease in mice: possible role of short-chain fatty acids and gut microbiota regulated by pectin.J Agric Food Chem. 2018;66:8015-25. https://doi.org/10.1021/acs.jafc.8b02979

47. Garcia-Mantrana I, Selma-Royo M, Alcantara C, Collado MC. Shifts on gut microbiota associated to mediterranean diet adherence and specific dietary intakes on general adult population. Front Microbiol. 2018;9:890. https://doi.org/10.3389/fmicb.2018.00890

48. Buckel W, Barker HA. Two pathways of glutamate fermentation by anaerobic bacteria. J Bacteriol. 1974;117:1248-60. https://doi.org/10.1128/jb.117.3.1248-1260.1974

49. Moser AM, Spindelboeck W, Halwachs B, Strohmaier H, Kump P, Gorkiewicz G, et al. Effects of an oral synbiotic on the gastrointestinal immune system and microbiota in patients with diarrhea-predominant irritable bowel syndrome. Eur J Nutr. 2019;58:2767-78. https://doi. org/10.1007/s00394-018-1826-7

50. Valeriano VDV, Balolong MP, Kang DK. Probiotic roles of Lactobacillus sp. in swine: insights from gut microbiota.J Appl Microbiol. 2017;122:554-67. https://doi.org/10.1111/jam.13364

51. Biddle A, Stewart L, Blanchard J, Leschine S. Untangling the genetic basis of fibrolytic specialization by Lachnospiraceae and Ruminococcaceae in diverse gut communities. Diversity. 2013;5:627-40. https://doi.org/10.3390/d5030627

52. Schnabl B, Brenner DA. Interactions between the intestinal microbiome and liver diseases. Gastroenterology. 2014;146:1513-24. https://doi.org/10.1053/j.gastro.2014.01.020

53. Zhuang M, Shang W, Ma Q, Strappe P, Zhou Z. Abundance of probiotics and butyrate-production microbiome manages constipation via short-chain fatty acids production and hormones secretion. Mol Nutr Food Res. 2019;63:1801187. https://doi.org/10.1002/mnfr.201801187 
54. Feng G, Mikkelsen D, Hoedt EC, Williams BA, Flanagan BM, Morrison M, et al. In vitro fermentation outcomes of arabinoxylan and galactoxyloglucan depend on fecal inoculum more than substrate chemistry. Food Funct. 2020;11:7892-904. https://doi.org/10.1039/ D0FO01103G

55. Ley RE. Prevotella in the gut: choose carefully. Nat Rev Gastroenterol Hepatol. 2016;13:6970. https://doi.org/10.1038/nrgastro.2016.4

56. De Filippo C, Cavalieri D, Di Paola M, Ramazzotti M, Poullet JB, Massart S, et al. Impact of diet in shaping gut microbiota revealed by a comparative study in children from Europe and rural Africa. Proc Natl Acad Sci USA. 2010;107:14691-6. https://doi.org/10.1073/ pnas. 1005963107

57. Hu R, Zeng F, Wu L, Wan X, Chen Y, Zhang J, et al. Fermented carrot juice attenuates type 2 diabetes by mediating gut microbiota in rats. Food Funct. 2019;10:2935-46. https://doi. org/10.1039/C9FO00475K

58. Li A, Ni W, Zhang Q, Li Y, Zhang X, Wu H, et al. Effect of cinnamon essential oil on gut microbiota in the mouse model of dextran sodium sulfate-induced colitis. Microbiol Immunol. 2020;64:23-32. https://doi.org/10.1111/1348-0421.12749

59. Pi Y, Hu J, Bai Y, Wang Z, Wu Y, Ye H, et al. Effects of dietary fibers with different physicochemical properties on fermentation kinetics and microbial composition by fecal inoculum from lactating sows in vitro. J Sci Food Agric. 2021;101:907-17. https://doi.org/10.1002/ jsfa.10698

60. Michail S, Lin M, Frey MR, Fanter R, Paliy O, Hilbush B, et al. Altered gut microbial energy and metabolism in children with non-alcoholic fatty liver disease. FEMS Microbiol Ecol. 2015;91:1-9. https://doi.org/10.1093/femsec/fiu002

61. Li J, Zhao F, Wang Y, Chen J, Tao J, Tian G, et al. Gut microbiota dysbiosis contributes to the development of hypertension. Microbiome. 2017;5:14. https://doi.org/10.1186/s40168-0160222-x

62. Wang C, Li W, Wang H, Ma Y, Zhao X, Zhang X, et al. Saccharomyces boulardii alleviates ulcerative colitis carcinogenesis in mice by reducing TNF- $\alpha$ and IL-6 levels and functions and by rebalancing intestinal microbiota. BMC Microbiol. 2019;19:246. https://doi.org/10.1186/ s12866-019-1610-8

63. Li F, Wang M, Wang J, Li R, Zhang Y. Alterations to the gut microbiota and their correlation with inflammatory factors in chronic kidney disease. Front Cell Infect Microbiol. 2019;9:206. https://doi.org/10.3389/fcimb.2019.00206

64. Kang X, Zhan L, Lu X, Song J, Zhong Y, Wang Y, et al. Characteristics of gastric microbiota in GK rats with spontaneous diabetes: a comparative study. Diabetes Metab Syndr Obes. 2020;13:1435-47. https://doi.org/10.2147/DMSO.S242698

65. Tamanai-Shacoori Z, Smida I, Bousarghin L, Loreal O, Meuric V, Fong SB, et al. Roseburia spp.: a marker of health? Future Microbiol. 2017;12:157-70. https://doi.org/10.2217/fmb2016-0130

66. Hua X, Zhu J, Yang T, Guo M, Li Q, Chen J, et al. The gut microbiota and associated metabolites are altered in sleep disorder of children with autism spectrum disorders. Front Psychiatry. 2020;11:855. https://doi.org/10.3389/fpsyt.2020.00855

67. Duncan SH, Holtrop G, Lobley GE, Calder AG, Stewart CS, Flint HJ. Contribution of acetate to butyrate formation by human faecal bacteria. Br J Nutr. 2004;91:915-23. https://doi. org/10.1079/BJN20041150

68. Morrison DJ, Mackay WG, Edwards CA, Preston T, Dodson B, Weaver LT. Butyrate production from oligofructose fermentation by the human faecal flora: what is the contribu- 
tion of extracellular acetate and lactate? Br J Nutr. 2006;96:570-7. https://doi.org/10.1079/ BJN20061853

69. Sun Y, O'Riordan MXD. Regulation of bacterial pathogenesis by intestinal short-chain fatty acids. Adv Appl Microbiol. 2013;85:93-118. https://doi.org/10.1016/B978-0-12-4076723.00003-4

70. Tsugawa H, Kabe Y, Kanai A, Sugiura Y, Hida S, Taniguchi S, et al. Short-chain fatty acids bind to apoptosis-associated speck-like protein to activate inflammasome complex to prevent Salmonella infection. PLOS Biol. 2020;18:e3000813. https://doi.org/10.1371/journal. pbio.3000813

71. Basen M, Kurrer SE. A close look at pentose metabolism of gut bacteria. FEBS J. 2021;288:1804-8. https://doi.org/10.1111/febs.15575

72. Tanca A, Abbondio M, Palomba A, Fraumene C, Manghina V, Cucca F, et al. Potential and active functions in the gut microbiota of a healthy human cohort. Microbiome. 2017;5:79. https://doi.org/10.1186/s40168-017-0293-3

73. Dai ZL, Wu G, Zhu WY. Amino acid metabolism in intestinal bacteria: links between gut ecology and host health. Front Biosci. 2011;16:1768-86. https://doi.org/10.2741/3820

74. Walsh CJ, Healy S, O’Toole PW, Murphy EF, Cotter PD. The probiotic L. casei LC-XCAL ${ }^{\mathrm{TM}}$ improves metabolic health in a diet-induced obesity mouse model without altering the microbiome. Gut Microbes. 2020;12:1747330. https://doi.org/10.1080/19490976.2020.1747330

75. Cao GT, Zhan XA, Zhang LL, Zeng XF, Chen AG, Yang CM. Modulation of broilers' caecal microflora and metabolites in response to a potential probiotic Bacillus amyloliquefaciens. J Anim Physiol Anim Nutr. 2018;102:e909-17. https://doi.org/10.1111/jpn.12856 